\title{
Bandwidth Enhancement of Antenna Arrays Utilizing Mutual Coupling between Antenna Elements
}

\author{
Min Wang, ${ }^{1}$ Wen Wu, ${ }^{1}$ and Zhongxiang Shen ${ }^{2}$ \\ ${ }^{1}$ Ministerial Key Laboratory of JGMT, Nanjing University of Science and Technology, Nanjing 210094, China \\ ${ }^{2}$ School of Electrical and Electronic Engineering, Nanyang Technological University, Singapore 639798 \\ Correspondence should be addressed to Min Wang, wangmin@mail.njust.edu.cn
}

Received 14 November 2009; Revised 26 January 2010; Accepted 31 March 2010

Academic Editor: Hoi Shun Lui

Copyright ( $) 2010$ Min Wang et al. This is an open access article distributed under the Creative Commons Attribution License, which permits unrestricted use, distribution, and reproduction in any medium, provided the original work is properly cited.

\begin{abstract}
The mutual coupling effect between antenna elements on an array's bandwidth is investigated using scattering parameters instead of the mutual impedance. First, an approximate expression is derived for matched voltage standing wave ratio (VSWR) bandwidth of a tuned antenna, which reveals that the bandwidth is inversely proportional to the magnitude $\left|\Gamma_{0}^{\prime}\left(\omega_{0}\right)\right|$ of the frequency derivative of the reflection coefficient. Next, considering linear antenna arrays with corporate feed as an example, closed-form expressions of the reflection coefficient are derived at the input port of the feeding network, which shows that the active reflection coefficient of an array is the linear superposition of elements' passive reflection coefficient $S_{11}$ and the mutual coupling coefficient $S_{12}$ from adjacent elements. The VSWR bandwidth expressions for an array imply that bandwidth enhancement of the overall array can be achieved when the element passive reflection coefficient $S_{11}$ and mutual coupling $S_{12}$ are cancelled, as well as the frequency derivatives $S_{11}^{\prime}$ and $S_{12}^{\prime}$ also cancel each other. Slot arrays and a two-element Vivaldi array are investigated to verify the validity of our theoretical analysis. Numerical and experimental results are presented to successfully demonstrate the bandwidth enhancement of antenna arrays utilizing mutual coupling effect.
\end{abstract}

\section{Introduction}

Antenna arrays are widely used in many practical systems to enhance gain or provide beam scanning capability. Mutual coupling between antenna elements is an important issue in designing antenna arrays. It modifies radiation pattern, beamwidth, and directivity of an array, and even degrades the performance of adaptive arrays [1-3]. However, Ludwig [1] demonstrated that mutual coupling is a natural and desirable effect in array behavior, and there is an optimum nonzero value of mutual coupling and passive reflection coefficient, which yields maximum array gain for an array in the active mode. It is also well known that the driving-point impedance of elements in an array with all elements excited depends upon the self-impedance, the mutual impedance, and input current relations between elements [4]. Yaghjian demonstrated that the matched voltage standing wave ratio (VSWR) bandwidth for an antenna tuned at a frequency $\omega_{0}$ is closely related to the input impedance of the antenna and its derivative [5]. Considering the driving-point impedance as the element's self-impedance loaded with the mutual impedance between elements, one can analyze mutual coupling effect on array bandwidth. However, the analysis associated with mutual impedance is usually quite complicated. In our previous work [6], we investigated mutual coupling effects on the performance of linear antenna array with corporate feed by analyzing arrays' active reflection coefficients, instead of the conventional mutual impedance. Obvious bandwidth enhancement by utilizing mutual coupling between elements had been experimentally demonstrated with printed slot arrays. In this paper, we improve both theoretical analysis and experimental research on mutual coupling effects on array bandwidth.

First, we derive an approximate expression for the matched VSWR bandwidth of a tuned antenna from the definition given in [5], which reveals that the bandwidth is inversely proportional to the magnitude $\left|\Gamma_{0}^{\prime}\left(\omega_{0}\right)\right|$ of the frequency derivative of the reflection coefficient. Then, the active reflection coefficient of a linear antenna arrays with corporate feed is investigated. For simplicity, the feeding 
network is assumed to be constructed by an equal-power divider with equal phases. Due to mutual coupling between array elements, conversion of energy implies unavoidable existence of cross-coupling between the feed lines [7]. Taking the array system as a cascaded connection of the array and the feeding network, closed-form expressions for the reflection coefficient at the feed input of the array can be derived. For a two-element array, the reflection coefficient is approximately the linear superposition of scattering coefficients $S_{11}$ and $S_{12}$ of the array when treated as a two-port network. For a linear array with all elements excited simultaneously, the active reflection coefficient is the linear superposition of elements' passive reflection coefficient and the mutual coupling from adjacent elements, which is similar to that of the array with two identical elements. Therefore, the mutual coupling effect on array bandwidth can be expressed in terms of $S$ parameters. The bandwidth expressions for an array imply that bandwidth enhancement of the overall array can be achieved when the element passive reflection coefficient $S_{11}$ and mutual coupling $S_{12}$ are cancelled, as well as the frequency derivatives $S_{11}^{\prime}$ and $S_{12}^{\prime}$ also cancel each other.

Finally, numerical simulation and experimental verification are carried out to verify the bandwidth enhancement. Printed slot antennas have extensive applications in phased arrays, satellite communication systems, and airborne systems due to their compact size and high efficiency [8-11] and are therefore considered here. Two- and four-element slot arrays are used to study their mutual coupling and to demonstrate the bandwidth enhancement by invoking suitable mutual coupling. Experimental prototypes are fabricated and measurement results verify the validity of our theoretical analysis. Furthermore, a two-element Vivaldi array has been investigated based on our formulation, which has a combination of two or more resonances and antiresonances. It is well known that performances of isolated Vivaldi antennas and elements in an array configuration differ greatly due to strong mutual coupling effect [12-15]. Particular parametric optimizations are conducted to obtain suitable mutual coupling and element's passive reflection coefficient for the array. Simulated results further demonstrate that appropriate mutual coupling between elements results in bandwidth enhancement and also provides a useful indicator for parametric optimization of Vivaldi arrays.

\section{Theoretical Analysis}

2.1. Matched VSWR Bandwidth. Yaghjian and Best investigated the bandwidth and $Q$ of a general single-feed (oneport) lossy or lossless linear antenna tuned to resonance or antiresonance and gave the formula for the fractional matched VSWR bandwidth $\mathrm{FBW}_{V}\left(\omega_{0}\right)$ [5]. Considering a general transmitting antenna composed of electromagnetically linear materials and fed by a feed line with characteristic impedance $Z_{\mathrm{ch}}$ that carried just one propagating mode and tuned at a frequency $\omega_{0}$, the tuned complex input impedance can be written as

$$
Z_{0}(\omega)=R_{0}(\omega)+j X_{0}(\omega)
$$

where the subscript notifies parameters for an antenna matched at $\omega_{0}$.

The reflection coefficient of the antenna is then

$$
\Gamma_{0}(\omega)=\frac{Z_{0}(\omega)-Z_{\mathrm{ch}}}{Z_{0}(\omega)+Z_{\mathrm{ch}}}
$$

The matched VSWR bandwidth for an antenna tuned at a frequency $\omega_{0}$ is defined as the difference between the two frequencies on either side of $\omega_{0}$ at which the VSWR equals a constant $s$ or, equivalently, at which $\left|\Gamma_{0}(\omega)\right|^{2}$ equals $\alpha=$ $(s-1)^{2} /(s+1)^{2}$. It is derived in [5] that the fractional matched VSWR bandwidth $\mathrm{FBW}_{V}\left(\omega_{0}\right)$ takes the form

$$
\operatorname{FBW}_{V}\left(\omega_{0}\right) \approx \frac{4 \sqrt{\beta} R_{0}\left(\omega_{0}\right)}{\omega_{0}\left|Z_{0}^{\prime}\left(\omega_{0}\right)\right|}, \quad \sqrt{\beta}=\sqrt{\frac{\alpha}{1-\alpha}}=\frac{s-1}{2 \sqrt{s}} \leq 1
$$

which holds for tuned antennas under the sufficient conditions that $X_{0}^{\prime}\left(\omega_{0}\right)$ and $R_{0}^{\prime}\left(\omega_{0}\right)$ do not change greatly over the bandwidth (conditions that hold if $\operatorname{FBW}_{V}\left(\omega_{0}\right) \ll 1$, which can always be satisfied if $\beta$ is chosen small enough).

In this paper, we would like to analyze mutual coupling effect on the bandwidth enhancement of antenna arrays. It is known that mutual coupling effect in an antenna array is commonly modeled as a change in the driving impedance of the elements and it is usually referred to as mutual impedance variation [4]. For the simplest array that consists of two identical elements with equal excitation, we can easily obtain the elements' driving-point impedances as follows:

$$
Z_{1 d}=Z_{11}+Z_{12}
$$

where $Z_{1 d}, Z_{11}$, and $Z_{12}$ represent the element's driving-point impedance, the element's self-impedance, and the mutual impedance, respectively.

For an array with all elements excited simultaneously, it is not the element's self-impedance but the driving-point impedance that should be matched. If the array is tuned at a frequency $\widetilde{\omega}_{0}$ (normally, $\widetilde{\omega}_{0}$ is not equal to the resonant or antiresonant frequency $\omega_{0}$ of elements due to mutual coupling effect), the conductance $X_{1 d 0}\left(\widetilde{\omega}_{0}\right)$ equals zero and the driving impedance is

$$
Z_{1 d 0}\left(\widetilde{\omega}_{0}\right)=Z_{110}\left(\widetilde{\omega}_{0}\right)+Z_{120}\left(\widetilde{\omega}_{0}\right)=R_{1 d 0}\left(\tilde{\omega}_{0}\right) .
$$

Substituting (5) into (3), we obtain the fractional matched VSWR bandwidth $\mathrm{FBW}_{V}\left(\widetilde{\omega}_{0}\right)$ for the array as

$$
\operatorname{FBW}_{V}\left(\tilde{\omega}_{0}\right) \approx \frac{4 \sqrt{\beta} R_{d 0}\left(\tilde{\omega}_{0}\right)}{\tilde{\omega}_{0}\left|Z_{d 0}^{\prime}\left(\tilde{\omega}_{0}\right)\right|}=\frac{4 \sqrt{\beta} R_{d 0}\left(\widetilde{\omega}_{0}\right)}{\widetilde{\omega}_{0}\left|Z_{110}^{\prime}\left(\widetilde{\omega}_{0}\right)+Z_{120}^{\prime}\left(\tilde{\omega}_{0}\right)\right|} .
$$

It is shown that from (3) and (6), provided we match the array with $R_{1 d 0}\left(\tilde{\omega}_{0}\right) \approx R_{0}\left(\omega_{0}\right)$ and appropriately tune impedances with $Z_{110}^{\prime}\left(\widetilde{\omega}_{0}\right) \approx-Z_{120}^{\prime}\left(\tilde{\omega}_{0}\right)$, the matched VSWR bandwidth $\operatorname{FBW}_{V}\left(\widetilde{\omega}_{0}\right)$ of the array would be greatly enhanced.

Through above analysis, we show that mutual coupling effect on array bandwidth could be analyzed in the view of 
the mutual impedance. However, the analysis associated with mutual impedance is usually quite complicated, especially for a larger array. The expressions of element's open-circuit voltage are usually needed to calculate the mutual impedance while array element's port voltage is difficult to be measured for most practical configurations or even to be defined in some situations. On the other hand, $S$-parameters are a versatile tool to analyze microwave networks. Furthermore, the matched VSWR bandwidth is naturally defined using the reflection coefficient. Therefore, we would rather investigate the mutual coupling effect on an array's bandwidth in the view of $S$-parameters. Therefore, we go back to the definition of matched VSWR bandwidth, $\left(\omega_{+}-\omega_{-}\right)$, which is determined by

$$
\left|\Gamma_{0}\left(\omega_{ \pm}\right)\right|^{2}=\alpha
$$

Expanding the left-hand side of (7) in a Taylor series about $\omega_{0}$, we find

$$
\left|\Gamma_{0}^{\prime}\left(\omega_{0}\right)\right|^{2}\left(\Delta \omega_{ \pm}\right)^{2} \approx \alpha
$$

under the condition $\Gamma_{0}\left(\omega_{0}\right) \approx 0$ and the assumption that the $O\left[\left(\Delta \omega_{ \pm}\right)^{3}\right]$ terms are negligible, where $\Gamma_{0}^{\prime}(\omega)$ denotes the frequency derivative of the reflection coefficient. This assumption is generally satisfied if $\left|\Delta \omega_{ \pm} / \omega\right| \ll 1$. The solutions to (8) for $\Delta \omega_{ \pm}$are

$$
\Delta \omega_{ \pm} \approx \pm \frac{\sqrt{\alpha}}{\left|\Gamma_{0}^{\prime}\left(\omega_{0}\right)\right|}
$$

which results in the fractional matched VSWR bandwidth $\mathrm{FBW}_{V}\left(\widetilde{\omega}_{0}\right)$

$$
\operatorname{FBW}_{V}\left(\omega_{0}\right)=\frac{\omega_{+}-\omega_{-}}{\omega_{0}}=\frac{\Delta \omega_{+}-\Delta \omega_{-}}{\omega_{0}} \approx \frac{2 \sqrt{\alpha}}{\omega_{0}\left|\Gamma_{0}^{\prime}\left(\omega_{0}\right)\right|}
$$

Equation (10) holds true for tuned antennas under the sufficient condition that $\Gamma_{0}^{\prime}(\omega)$ does not change greatly over the bandwidth. It also reveals that the fractional matched VSWR bandwidth for a tuned antenna is inversely proportional to the absolute value of the derivative of the reflection coefficient with respect to $\omega$. Note that (10) is a general equation for antennas that can be used to investigate mutual coupling effect on bandwidth in terms of $S$-parameters.

2.2. Active Reflection Coefficient of Arrays. Figure 1 shows a two-element array fed through a 3 -dB T-branch power divider, which can be considered as the cascaded connection of a three-port network (T-branch power divider) with a two-port network. The incident and reflected waves are labelled at the ports as $a, a_{1}, a_{2}, b, b_{1}$, and $b_{2}$. The relationships between incident and reflected waves can be established by the $S$-matrix of these two networks, so that the reflection coefficient of the overall network is derived.
For a lossless 3-dB T-branch power divider [15], we know that

$$
\left[\begin{array}{c}
b \\
a_{1} \\
a_{2}
\end{array}\right]=\left[\begin{array}{ccc}
0 & \frac{1}{\sqrt{2}} & \frac{1}{\sqrt{2}} \\
\frac{1}{\sqrt{2}} & -\frac{1}{2} & \frac{1}{2} \\
\frac{1}{\sqrt{2}} & \frac{1}{2} & -\frac{1}{2}
\end{array}\right]\left[\begin{array}{l}
a \\
b_{1} \\
b_{2}
\end{array}\right] .
$$

For the antenna array, we consider only passive and identical elements, and then we have

$$
\left[\begin{array}{l}
b_{1} \\
b_{2}
\end{array}\right]=\left[\begin{array}{ll}
S_{11} & S_{12} \\
S_{12} & S_{11}
\end{array}\right]\left[\begin{array}{l}
a_{1} \\
a_{2}
\end{array}\right]
$$

Due to the symmetry of the array and the power divider, it can be easily derived from (11) and (12) that

$$
\begin{gathered}
a_{1}=a_{2}=\frac{1}{\sqrt{2}} a, \\
b_{1}=b_{2}=\frac{1}{\sqrt{2}} a\left(S_{11}+S_{12}\right) .
\end{gathered}
$$

Finally, we obtain the reflection coefficient of the overall network at the input port of the divider as

$$
\Gamma=\frac{b}{a}=S_{11}+S_{12} .
$$

Equation (14) shows that the reflection coefficient $\Gamma$ of the overall network is the superposition of $S_{11}$ and $S_{12}$ for the two-antenna array.

We further discuss a linear array with 4 identical elements fed through a 4-way power divider which is shown in Figure 2, with the incident and reflected waves labeled at the ports. Similarly, considering the system as the cascaded connection of a five-port network (power divider) with a four-port network, we can write the relationship of those input and output signals as

$$
\begin{aligned}
& {\left[\begin{array}{l}
b \\
a_{1} \\
a_{2} \\
a_{3} \\
a_{4}
\end{array}\right]=\left[\begin{array}{ccccc}
0 & \frac{1}{2} & \frac{1}{2} & \frac{1}{2} & \frac{1}{2} \\
\frac{1}{2} & -\frac{3}{4} & \frac{1}{4} & \frac{1}{4} & \frac{1}{4} \\
\frac{1}{2} & \frac{1}{4} & -\frac{3}{4} & \frac{1}{4} & \frac{1}{4} \\
\frac{1}{2} & \frac{1}{4} & \frac{1}{4} & -\frac{3}{4} & \frac{1}{4} \\
\frac{1}{2} & \frac{1}{4} & \frac{1}{4} & \frac{1}{4} & -\frac{3}{4}
\end{array}\right]\left[\begin{array}{l}
a \\
b_{1} \\
b_{2} \\
b_{3} \\
b_{4}
\end{array}\right],} \\
& {\left[\begin{array}{l}
b_{1} \\
b_{2} \\
b_{3} \\
b_{4}
\end{array}\right]=\left[\begin{array}{cccc}
S_{11} & S_{12} & 0 & 0 \\
S_{12} & S_{22} & S_{23} & 0 \\
0 & S_{23} & S_{22} & S_{12} \\
0 & 0 & S_{12} & S_{11}
\end{array}\right]\left[\begin{array}{l}
a_{1} \\
a_{2} \\
a_{3} \\
a_{4}
\end{array}\right] .}
\end{aligned}
$$

The fact that the array is symmetric and array elements are identical is considered in (16), and only the mutual coupling between adjacent elements is taken into account. 


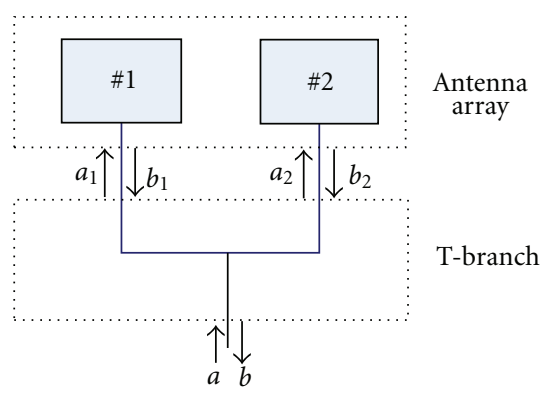

Figure 1: A two-element array fed through a T-branch power divider.

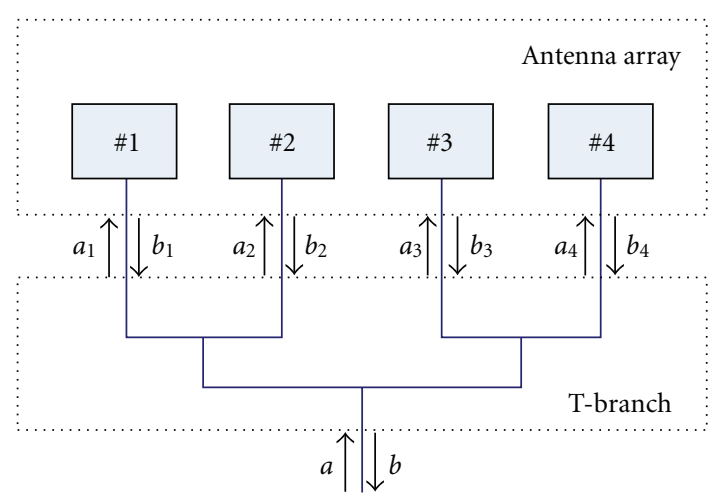

FIgURE 2: A four-element array fed through a 4-way power divider.

Because $a_{1}=a_{4}, a_{2}=a_{3}$, and $b_{1}=b_{4}, b_{2}=b_{3},(15)$ and (16) are simplified as

$$
\begin{aligned}
& {\left[\begin{array}{l}
b \\
a_{1} \\
a_{2}
\end{array}\right]=\left[\begin{array}{ccc}
0 & 1 & 1 \\
\frac{1}{2} & -\frac{1}{2} & \frac{1}{2} \\
\frac{1}{2} & \frac{1}{2} & -\frac{1}{2}
\end{array}\right]\left[\begin{array}{l}
a \\
b_{1} \\
b_{2}
\end{array}\right],} \\
& {\left[\begin{array}{l}
b_{1} \\
b_{2}
\end{array}\right]=\left[\begin{array}{cc}
S_{11} & S_{12} \\
S_{12} & S_{22}+S_{23}
\end{array}\right]\left[\begin{array}{l}
a_{1} \\
a_{2}
\end{array}\right] .}
\end{aligned}
$$

Let $S_{22}^{\prime}=S_{22}+S_{23}$, then we know that

$$
\begin{aligned}
& a_{1}=\frac{1+S_{12}-S_{22}^{\prime}}{2+S_{11}-S_{22}^{\prime}} a, \\
& a_{2}=\frac{1+S_{11}-S_{12}}{2+S_{11}-S_{22}^{\prime}} a .
\end{aligned}
$$

Finally, the reflection coefficient of the overall array is derived as

$$
\Gamma=\frac{b}{a}=2 S_{12}+\frac{S_{11}+S_{22}+S_{23}-2 S_{12}}{2+S_{11}-S_{22}-S_{23}} .
$$

Assuming that $S_{11}=S_{22}, S_{12}=S_{23},(19)$ is approximated for small value of $S_{12}$

$$
\Gamma \simeq 2 S_{12}+\frac{2 S_{11}-S_{12}}{2-S_{12}} \simeq S_{11}+\frac{3}{2} S_{12} .
$$

Equation (20) shows the similar property as (14) that the reflection coefficient of the array fed through a corporate feed is the linear superposition of $S_{11}$ and $S_{12}$, which can be generalized to the active reflection coefficient of the $\mathrm{N}$ element linear array with all elements excited simultaneously.

Generally, we assume all the elements to be fed with equal powers, $a_{1}=a_{2}=\cdots=a_{N}=a / \sqrt{N}$, all the reflection coefficients of antenna ports to be equal, $S_{11}=S_{22}=\cdots=$ $S_{N N}$, the mutual coupling between the adjacent elements to be equal, $S_{12}=S_{23}=\cdots=S_{N-1, N}$, and all the other mutual coupling to be neglected. Under these assumptions, we get

$$
b_{i} \approx \begin{cases}\frac{\left(S_{11}+S_{12}\right) a}{\sqrt{N}} & i=1, N \\ \frac{\left(S_{11}+2 S_{12}\right) a}{\sqrt{N}} & \text { elsewhere. }\end{cases}
$$

The active reflection coefficient of the overall array is derived as

$$
\begin{aligned}
\Gamma & =\frac{b}{a} \\
& \approx \frac{\sum_{i=1}^{N} b_{i} / \sqrt{N}}{a} \\
& \approx S_{11}+\frac{2(N-1)}{N} S_{12} \\
& \approx S_{11}+2 S_{12} \quad(N \text { is large }) .
\end{aligned}
$$

Equation (22) gives a general closed-form expression for the active reflection coefficient of the $\mathrm{N}$-element linear array, which keeps the form of linear superposition of element passive reflection coefficient $S_{11}$ and mutual coupling $S_{12}$.

2.3. Mutual Coupling Effect on Array Bandwidth. Taking an $\mathrm{N}$-element linear array fed through a corporate feed as a single port antenna, it can be known from (22) that the array could be tuned at a frequency $\widetilde{\omega}_{0}$ where

$$
S_{11}\left(\widetilde{\omega}_{0}\right) \approx-\frac{2(N-1)}{N} S_{12}\left(\widetilde{\omega}_{0}\right)
$$

and $\Gamma\left(\widetilde{\omega}_{0}\right)=0$. Due to the mutual coupling effect, $\widetilde{\omega}_{0}$ is not equal to the resonant or antiresonant frequency [5] of the single elements $\omega_{0}$, but normally $\left|\left(\widetilde{\omega}_{0}-\omega_{0}\right) / \omega_{0}\right| \ll 1$.

We substitute (22) into (10) to obtain the matched VSWR bandwidth for the array at a given $\alpha$ as

$$
\begin{aligned}
\operatorname{FBW}_{V}\left(\widetilde{\omega}_{0}\right) & \approx \frac{2 \sqrt{\alpha}}{\widetilde{\omega}_{0}\left|\Gamma^{\prime}\left(\widetilde{\omega}_{0}\right)\right|} \\
& \approx \frac{2 \sqrt{\alpha}}{\widetilde{\omega}_{0}\left|S_{11}^{\prime}\left(\widetilde{\omega}_{0}\right)+(2 N-1) S_{12}^{\prime}\left(\widetilde{\omega}_{0}\right) / N\right|} .
\end{aligned}
$$

For a single element, $S_{11}^{\prime}(\omega)$ does not change greatly nearby $\omega_{0}$. Therefore, when comparing (24) and (10), we know that the bandwidth of the overall array system should be affected by the relation between derivatives of element passive reflection coefficient $S_{11}$ and mutual coupling $S_{12}$.

Equations (23) and (24) imply two conditions which should be satisfied to achieve bandwidth enhancement 


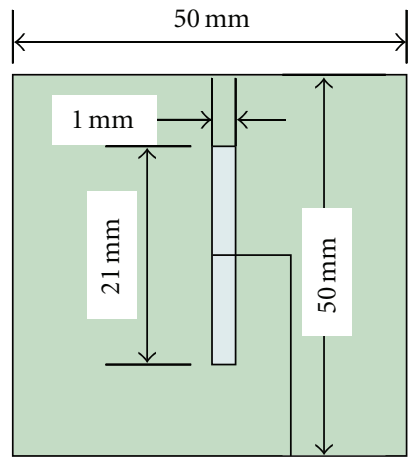

(a) Single slot antenna

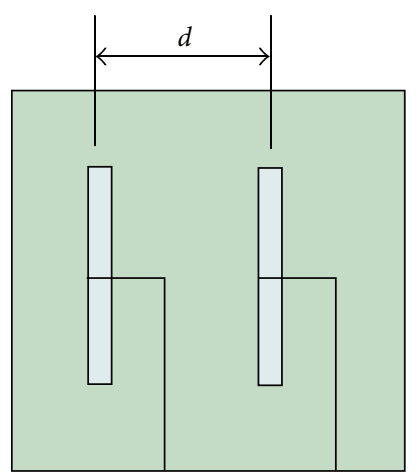

(b) Array no. 1

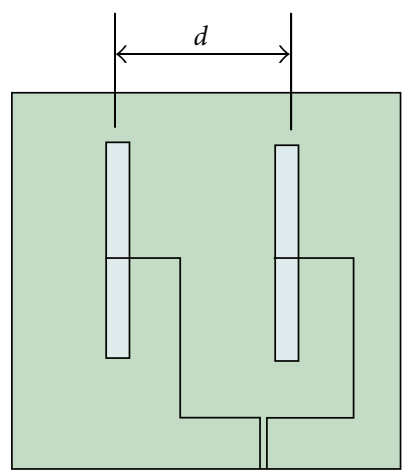

(c) Array no. 2

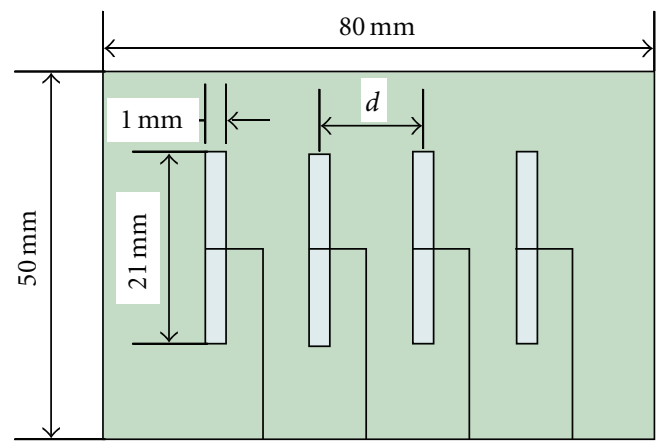

(d) Array no. 3

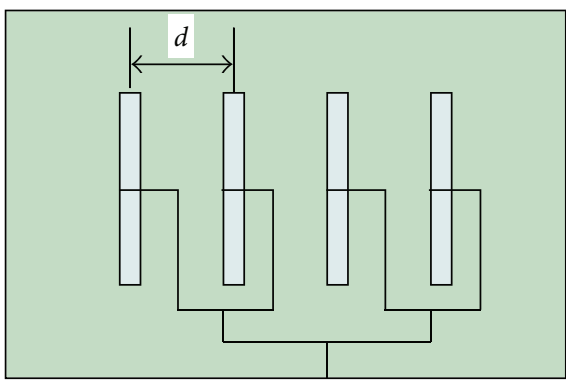

(e) Array no. 4

Figure 3: The layout of slot antennas.

for the whole array. Firstly, the element passive reflection coefficient $S_{11}$ and mutual coupling $S_{12}$ should be out of phase and be cancelled as shown in (23), so that the array can be tuned. Furthermore, the frequency derivatives $S_{11}^{\prime}$ and $S_{12}^{\prime}$ should be out of phase to cancel each other. We will conduct numerical simulation and experimental verification to demonstrate this bandwidth enhancement.

\section{Simulated and Measured Results}

The closed-form expressions derived in the previous section imply that the bandwidth enhancement of the overall array can be achieved by invoking appropriate mutual coupling between elements. In this section, numerical simulation and experimental verification are conducted to investigate mutual coupling effect on array bandwidth. Two types of antenna are underanalysed. Firstly, we consider slot antenna arrays, which are normally narrowband and only have one antiresonant frequency. Then, a broadband two-element Vivaldi array is investigated, which has a combination of two or more resonances and antiresonances that are so close together to build a broad bandwidth. To optimize the array bandwidth, we will vary element spacing $d$ to match the active array with appropriate amplitudes and phase differences for $S_{11}$ and $S_{12}$ as shown in (23). Then, for a given element spacing $d$, particular parametric optimizations are conducted to achieve bandwidth enhancement for the arrays by cancelling the derivatives of $S$-parameters as shown in (24).
3.1. Slot Antenna Array. We consider a single-slot antenna and an array of two identical slots shown in Figure 3. Their resonant frequencies are about $5.8 \mathrm{GHz}$. The substrate used is Rogers RO4230 $\left(\varepsilon_{r}=3\right)$ of thickness $0.762 \mathrm{~mm}$. The slots are cut on the ground plane of the substrate and the microstrip feed lines are printed on the top side. Array no. 1, which is fed through two independent microstrip lines, is used to obtain $S$-parameters of the array. Array no. 2, which is fed through a T-branch power divider, is the one we hope to investigate.

Obviously, it is the element spacing $d$ that mainly influences the mutual coupling between elements. So the phase differences between $S_{11}$ and $S_{12}$ of array no. 1 with different spacings are firstly calculated and shown in Figure 4 . Note that the element spacing should be less than one wavelength to avoid grating lobes and evoke mutual coupling strong enough to achieve bandwidth enhancement. Figure 4 indicates that the phase difference between $S_{11}$ and $S_{12}$ changes with the element spacing and has a sharp turn at the resonant frequency. Therefore, we can evaluate the spacing to achieve appropriate amplitudes and phase difference of $\pm \pi$ for $S$ parameters approximately at a small deviation from the resonant frequency, meanwhile, physical parameters are optimized to ensure proper phase relation for their derivatives with respect to frequency. In particular, we found the optimized spacing $d=14 \mathrm{~mm}$ (about a quarter wavelengths at the resonant frequency), as well as the optimized physical parameters, with which the bandwidth of the antenna array obviously improved. The corresponding simulated results for phase difference 


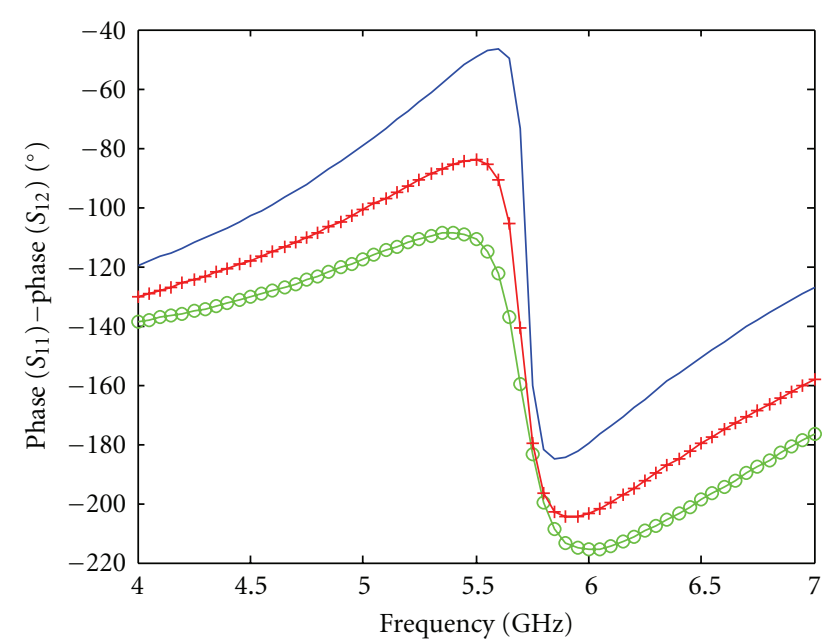

$\begin{aligned}- & d=10 \mathrm{~mm} \\ + & d=15 \mathrm{~mm} \\ -d & =20 \mathrm{~mm}\end{aligned}$

FIgURE 4: Calculated phase differences between $S_{11}$ and $S_{12}$ of array no. 1 .

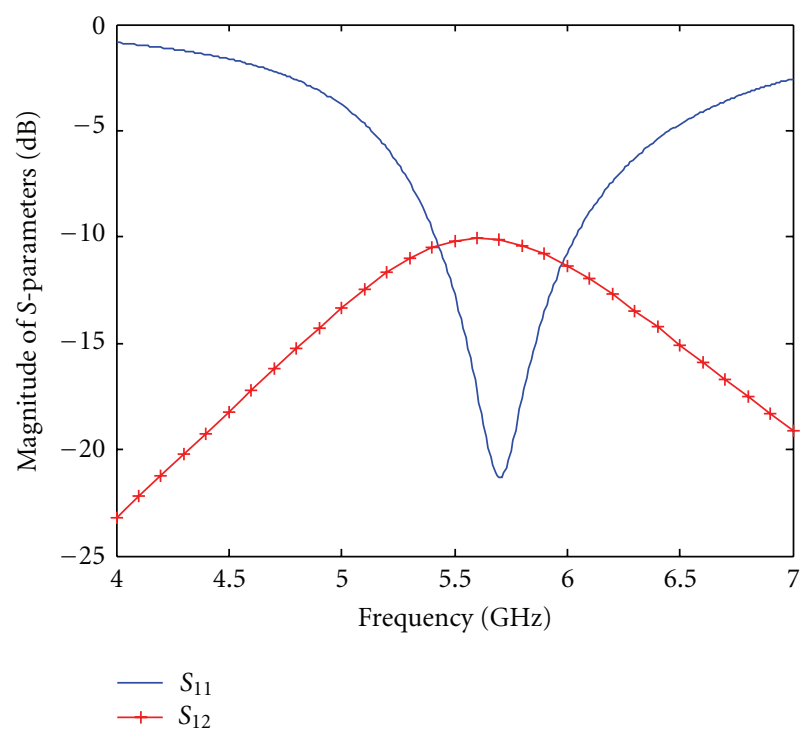

Figure 5: Simulated magnitudes of $S_{11}$ and $S_{12}$ of array no. 1 .

and magnitude of $S$ parameter are shown in Figures 4 and 5 , respectively. It can be seen that $S_{11}$ and $S_{12}$ are approximately equal in magnitude and opposite in phase near the frequency of $5.98 \mathrm{GHz}$, so that the active array can be expected to be tuned near this frequency. Figure 6 shows the optimized results of phase difference between derivatives of $S$ parameters, which show that phase differences about $\pm \pi$ have been obtained to strive for bandwidth enhancement of array. The single antenna and array no. 2 are designed and fabricated to verify our analysis, and simulated and measured results of return loss are shown in Figure 7. We can see that the resonant frequency of the array $\widetilde{\omega}_{0}=$ $5.98 \mathrm{GHz}$ is slightly different from that of the single slot

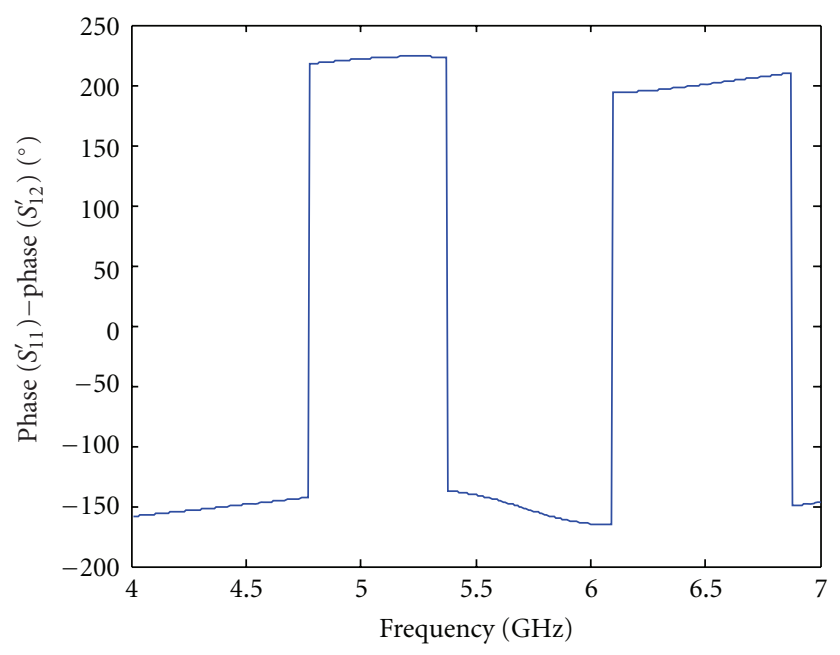

FIGURE 6: Calculated phase differences between $S_{11}^{\prime}$ and $S_{12}^{\prime}$ of array no. 1.

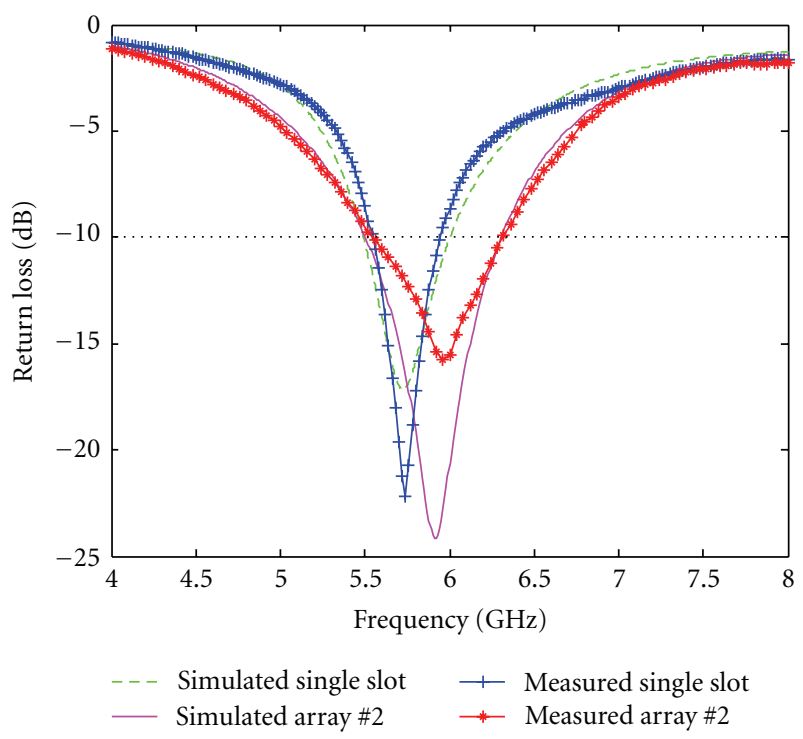

FIGURE 7: Simulated and measured return loss results of single-slot and two-slot array no. $2(d=14 \mathrm{~mm})$.

$\omega_{0}=5.70 \mathrm{GHz}$, just as what we analysed above. It should be more emphasised that obviously bandwidth enhancements have been demonstrated. It is seen from Figure 7 that the bandwidth is improved from $0.50 \mathrm{GHz}(6.00 \mathrm{GHz}-$ $5.50 \mathrm{GHz})$ to $0.80 \mathrm{GHz}(6.30 \mathrm{GHz}-5.50 \mathrm{GHz})$ according to simulated results as well as from $0.40 \mathrm{GHz}(5.95 \mathrm{GHz}-$ $5.55 \mathrm{GHz})$ to $0.76 \mathrm{GHz}(6.31 \mathrm{GHz}-5.55 \mathrm{GHz})$ according to measured ones. Measured results are in very good agreement with simulated ones. Moreover, in order to evaluate mutual coupling effect on array performance in general, we further provide realized gain results of a single-slot antenna and array no. 2, which are shown in Figure 8. We can see that moderately improved gain results have been achieved as well as the bandwidth enhancement. 


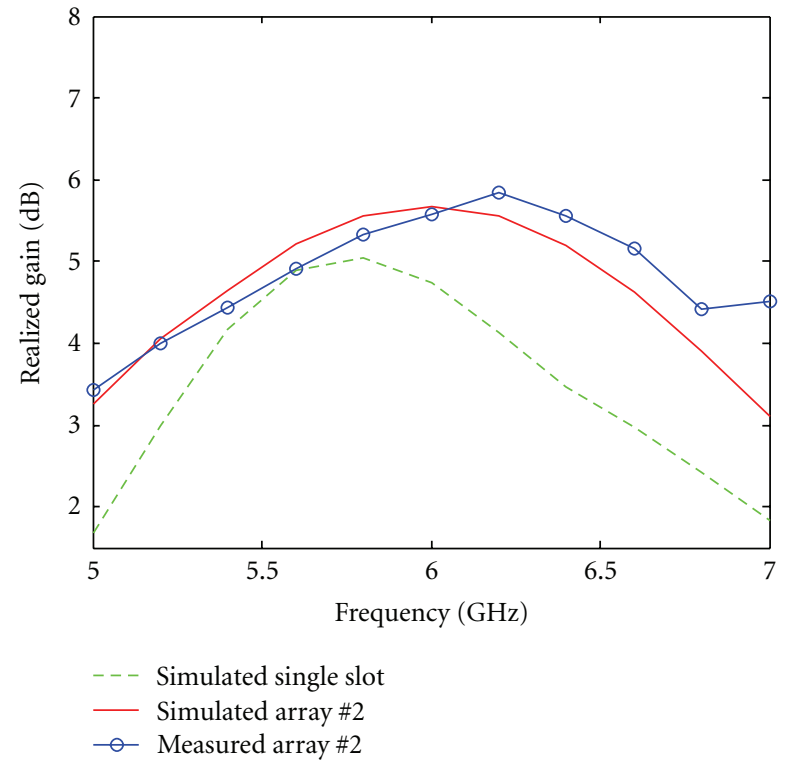

FIGURE 8: Simulated and measured gain results of single-slot and two-slot array no. $2(d=14 \mathrm{~mm})$.

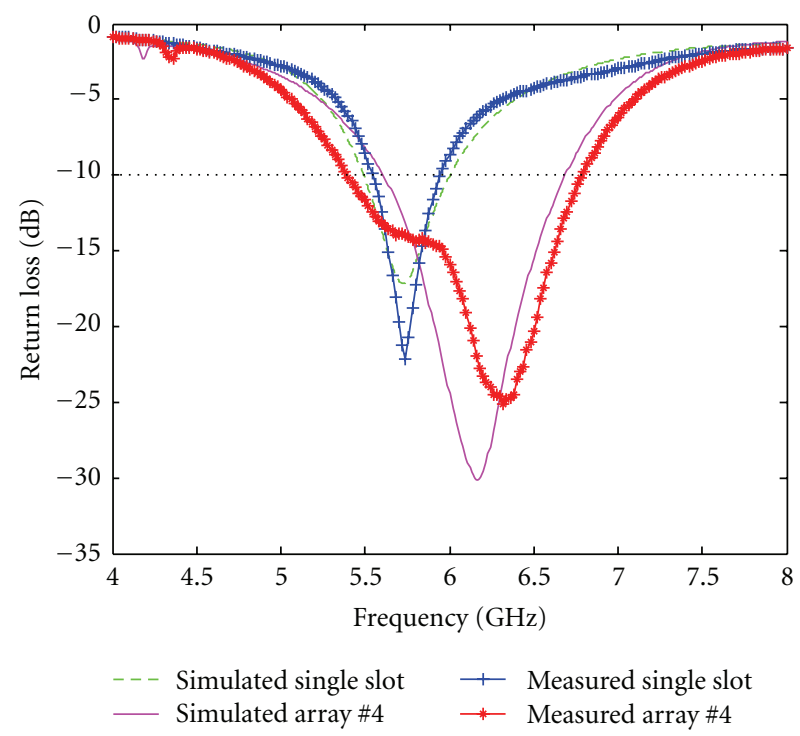

FIGURE 9: Simulated and measured return loss results of single-slot and four-slot array no. $4(d=14 \mathrm{~mm})$.

Next, we consider arrays consisting of four identical slots shown in Figure 3 to further demonstrate our analysis. Array no. 3 that is fed by four independent microstrip lines is used to obtain the $S$-parameters of the array. Array no. 4 fed through T-branch power dividers is the one we hope to investigate. The same substrate and element dimension are adopted as the two-element slot array. Particularly, we evaluate the optimized spacing $d=14 \mathrm{~mm}$ again for approximately achieving optimal $S$ parameters and their derivatives. The comparison of return loss results between a single-slot antenna and the four-slot array no. 4 is shown in Figure 9. It is seen that the bandwidth

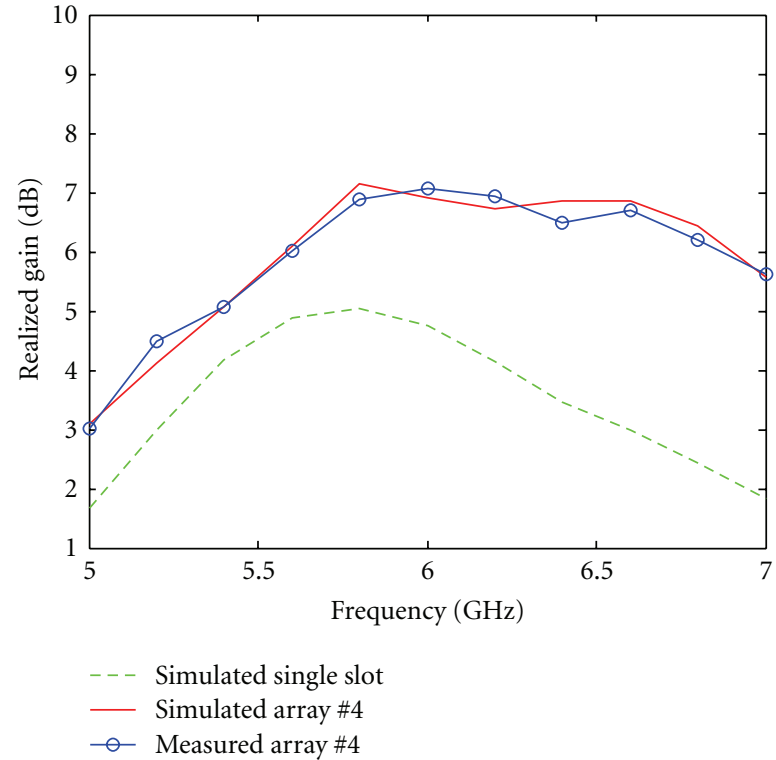

FIGURE 10: Simulated and measured gain results of single-slot and four-slot array no. $4(d=14 \mathrm{~mm})$.

of array no. 4 is improved from $0.50 \mathrm{GHz}(6.00 \mathrm{GHz}-$ $5.50 \mathrm{GHz})$ to $1.09 \mathrm{GHz}(6.69 \mathrm{GHz}-5.60 \mathrm{GHz})$ according to simulated results and from $0.40 \mathrm{GHz}(5.95 \mathrm{GHz}-5.55 \mathrm{GHz})$ to $1.38 \mathrm{GHz}(6.78 \mathrm{GHz}-5.40 \mathrm{GHz})$ according to measured ones. The realized gain results are also provided in Figure 10, which show that moderately improved gain results have been achieved as well as the bandwidth enhancement.

3.2. Vivaldi Antenna Array. The tapered slot antenna (TSA) was initially introduced as an array element by Lewis et al. in 1974 [11]. Gibson [12] later named the exponentially tapered slot antenna as Vivaldi antenna. Because of its potential for wide-band and wide-scan arrays, ease of fabrication, and convenient feed techniques, Vivaldi antenna array is a prime candidate for high-performance phased-array systems. In this paper, Vivaldi array is chosen as an example for broadband arrays having a combination of two or more resonances and antiresonances $\omega_{1}, \omega_{2}, \ldots, \omega_{n}, \ldots, \omega_{N}(N \geq$ 2 ). The bandwidth enhancement can be achieved at these frequencies by utilizing mutual coupling between array elements, so that the total bandwidth can be improved for a broadband array.

We consider a two-element Vivaldi array shown in Figure 11. For each element, the stripline feed is coupled to bilateral slotline cut on the ground plane. Element parameters can be subdivided into the stripline/slotline transition, the tapered slot, and the stripline stub and slotline cavity [13]. The bilateral slotline is terminated at one end with a circular slot cavity of diameter $D_{\mathrm{SL}}$, and at the other end it opens into an exponentially tapered slotline with an opening rate of $R$. The stripline feed is terminated in a radial line stub with radius $R_{\mathrm{ST}}$ and angle $\theta$. The stripline/slotline transition is specified by $W_{\mathrm{ST}}$ (stripline width) and $W_{\mathrm{SL}}$ (slotline width). Distances from the transition to the slotline cavity 


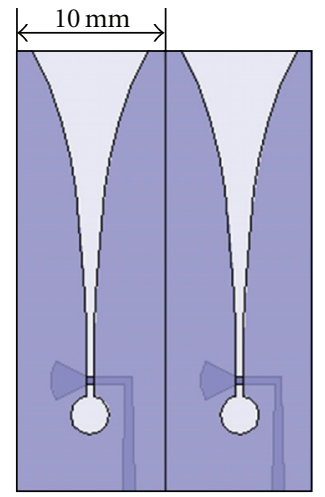

(a) A two-element Vivaldi array

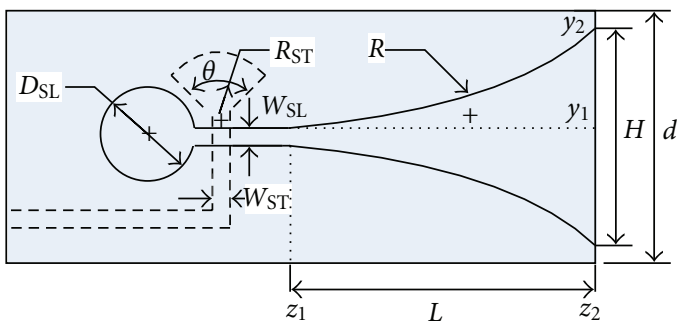

(b) Definition of element configuration

FIgURE 11: Schematic graph of Vivaldi antenna array.

and the taper can be properly adjusted to accommodate the transition. Broadband behavior has been obtained by appropriately designed $W_{\mathrm{ST}}, W_{\mathrm{SL}}, D_{\mathrm{SL}}, R_{\mathrm{ST}}$, and $\theta$ with coupling from the dominant stripline mode to the traveling wave in tapered slot. The broadband characteristic of antenna itself mainly attributes to the exponential taper profile, which is defined by the opening rate $R$ and two points $P_{1}\left(z_{1}, y_{1}\right)$ and $P_{2}\left(z_{2}, y_{2}\right)$

$$
y=c_{1} e^{R z}+c_{2}
$$

where

$$
c_{1}=\frac{y_{2}-y_{1}}{e^{R z_{2}}-e^{R z_{1}}}, \quad c_{2}=\frac{y_{1} e^{R z_{2}}-y_{2} e^{R z_{1}}}{e^{R z_{2}}-e^{R z_{1}}} .
$$

The taper length $L$ is $z_{2}-z_{1}$ and the aperture height $H$ is $2\left(y_{2}-y_{1}\right)+W_{\text {SL }}$.

The Vivaldi antenna element has theoretically unlimited bandwidth, and the upper operating frequency is mainly limited by the transition. However, for a Vivaldi array, the upper operating frequency is limited by the onset of grating lobes, which is determined by the element spacing. Therefore, we usually prescribe the element spacing and optimize antenna parameters to achieve desired bandwidth of the array. When the element spacing is prescribed, the mutual coupling between elements $S_{12}$ varies slightly and the bandwidth enhancement is mainly achieved by optimizing the elements' passive reflection coefficient $S_{11}$ to be well cancelled with $S_{12}$.

Here, we prescribe the element spacing $d=10 \mathrm{~mm}$, which is also the element width. The substrate used is

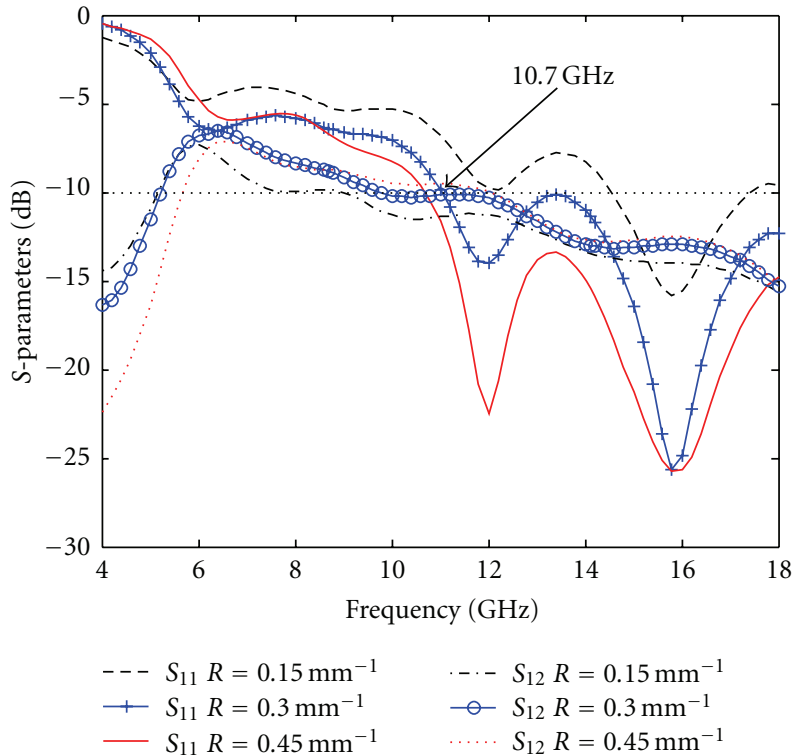

FIGURE 12: $S$-parameters with various opening rates $R$.

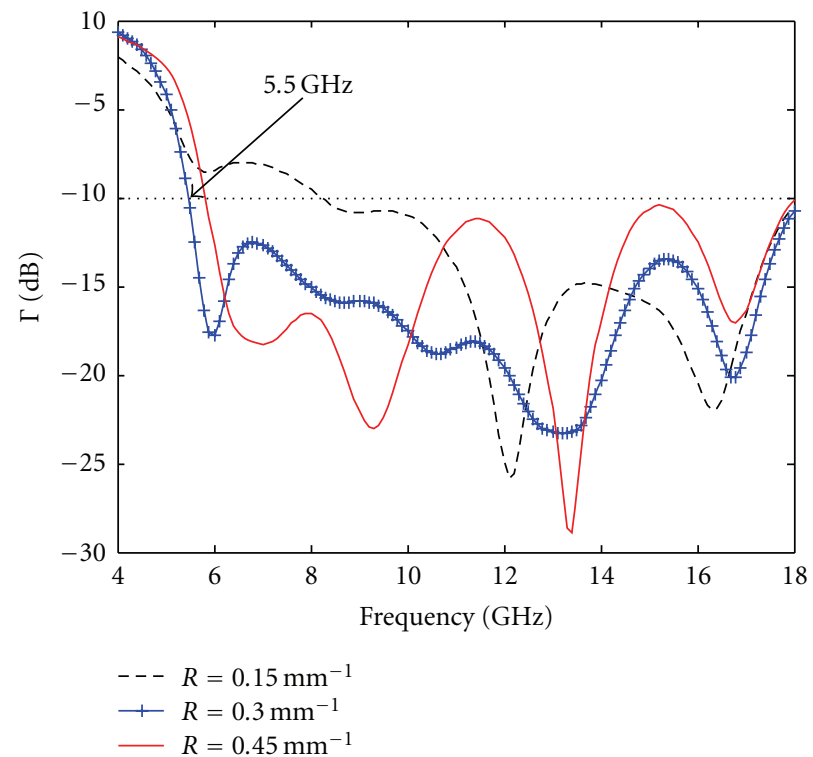

FIGURE 13: The active reflection coefficient $\Gamma$ with various opening rates $R$.

RO4230 $\left(\varepsilon_{r}=3\right)$ of thickness $1.524 \mathrm{~mm}$. After some optimization following $[14,15]$, the physical parameters are chosen: $H=8 \mathrm{~mm}, W_{\mathrm{ST}}=W_{\mathrm{SL}}=0.5 \mathrm{~mm}, D_{\mathrm{SL}}=R_{\mathrm{ST}}=$ $2.6 \mathrm{~mm}, \theta=70^{\circ}, L=22 \mathrm{~mm}$, and $R=0.3 \mathrm{~mm}^{-1}$. To clarify the relationship between $S_{11}$ and $S_{12}$, the variations of $S$-parameters with respect to different opening rates $R$ are provided and are shown in Figure 12. The corresponding active reflection coefficients $\Gamma$ are shown in Figure 13. It is shown that with $R=0.3 \mathrm{~mm}^{-1}$, the minimum operating frequency is about $5.5 \mathrm{GHz}$ while the frequency of one element is about $10.7 \mathrm{GHz}$. For a specified upper operating 


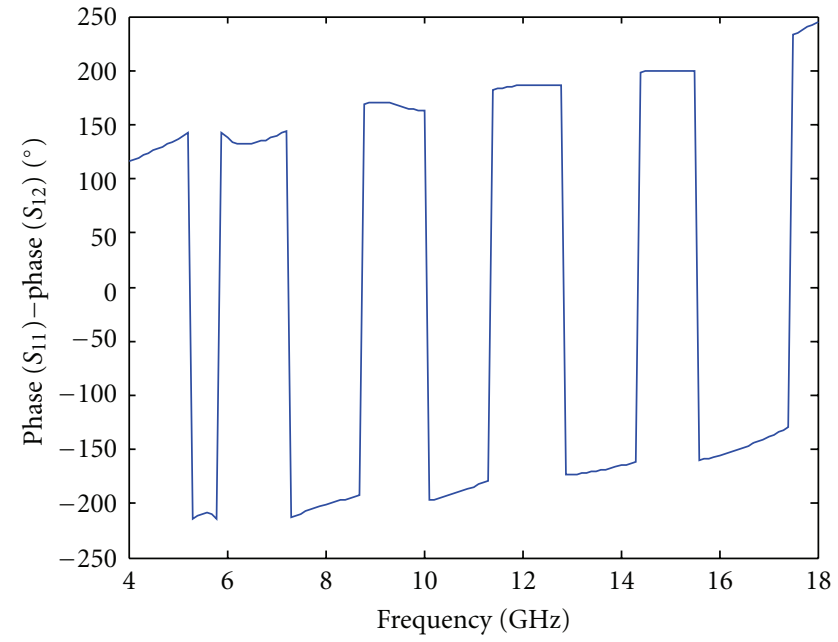

FIGURE 14: The phase difference between $S_{11}$ and $S_{12}$ with $R=$ $0.3 \mathrm{~mm}^{-1}$.

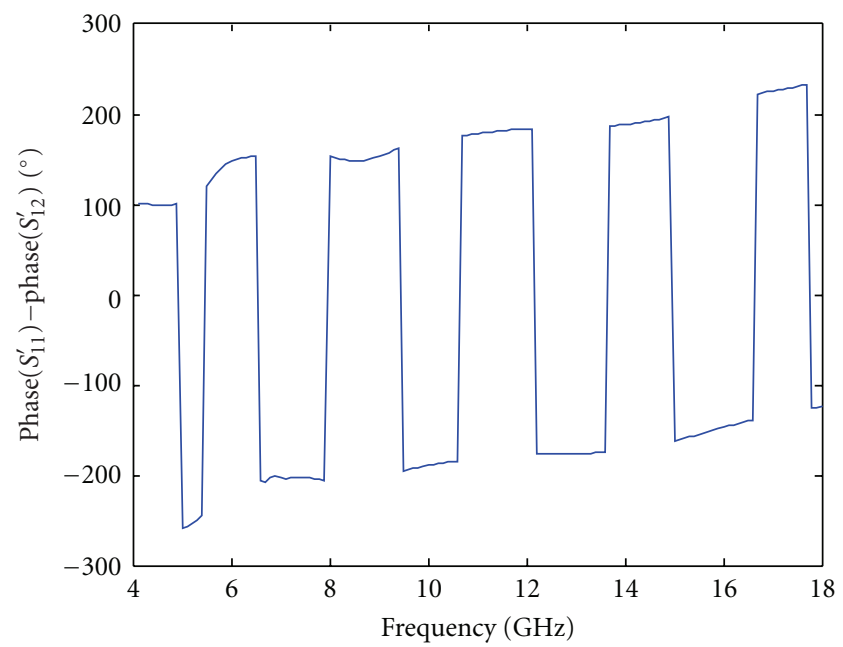

FIGURE 15: The phase difference between $S_{11}^{\prime}$ and $S_{12}^{\prime}$ with $R=$ $0.3 \mathrm{~mm}^{-1}$.

frequency $18 \mathrm{GHz}$, an optimum 3:1 bandwidth of the array has been achieved. Figure 14 shows the phase difference between $S_{11}$ and $S_{12}$ with $R=0.3 \mathrm{~mm}^{-1}$, and Figure 15 shows the phase difference between $S_{11}^{\prime}$ and $S_{12}^{\prime}$, which are all about $\pm \pi$. Simulation results show that the bandwidth of the Vivaldi array has been successfully improved by properly adjusting the passive reflection coefficient of elements along with mutual coupling. The phase differences between $S_{11}$ and $S_{12}$ and between $S_{11}^{\prime}$ and $S_{12}^{\prime}$ can also be useful indicators for design of Vivaldi array.

\section{Conclusions}

In this paper, mutual coupling between array elements has been utilized to achieve bandwidth enhancement based on the formulations for the matched VSWR bandwidth and the reflection coefficient of arrays with corporate feed. Instead of sticking to analysis of the mutual impedance, the active reflection coefficient of the array has been investigated to directly guide the optimization of bandwidth. Our theoretical analyses show that bandwidth enhancement of the overall array can be achieved when the element passive reflection coefficient $S_{11}$ and mutual coupling $S_{12}$ are well cancelled, as well as the frequency derivatives $S_{11}^{\prime}$ and $S_{12}^{\prime}$ also cancel each other. Both numerical simulated and experimental results successfully demonstrate our analysis.

\section{References}

[1] A. C. Ludwig, "Mutual coupling, gain, and directivity of an array of two identical antennas," IEEE Transactions on Antennas and Propagation, vol. 24, no. 6, pp. 837-841, 1976.

[2] I. J. Gupta and A. A. Ksienski, "Effect of mutual coupling on the performance of adaptive arrays," IEEE Transactions on Antennas and Propagation, vol. 31, no. 5, pp. 785-791, 1983.

[3] J. A. G. Malherbe, "Analysis of a linear antenna array including the effects of mutual coupling," IEEE Transactions on Education, vol. 32, no. 1, pp. 29-34, 1989.

[4] C. A. Balanis, Antenna Theory-Analysis and Design, John Wiley \& Sons, New York, NY, USA, 3rd edition, 2005.

[5] A. D. Yaghjian and S. R. Best, "Impedance, bandwidth, and Q of antennas," IEEE Transactions on Antennas and Propagation, vol. 53, no. 4, pp. 1298-1324, 2005.

[6] M. Wang and Z. Shen, "Mutual coupling effect on the performance of antenna arrays with corporate feed," in Proceedings of Asia-Pacific Symposium on Electromagnetic Compatibility and 19th International Zurich Symposium on Electromagnetic Compatibility (APEMC '08), pp. 546-549, Singapore, 2008.

[7] S. Stein, "On cross coupling in multiple-beam antennas," IRE Transactions on Antennas and Propagation, vol. 10, no. 5, pp. 548-557, 1962.

[8] Q. Li and Z. Shen, "Inverted microstrip-fed cavity-backed slot antennas," IEEE Antennas and Wireless Propagation Letters, vol. 1, pp. 98-101, 2002.

[9] S. I. Latif, L. Shafai, and S. K. Sharma, "Bandwidth enhancement and size reduction of microstrip slot antennas," IEEE Transactions on Antennas and Propagation, vol. 53, no. 3, pp. 994-1003, 2005.

[10] R. Garg, P. Bhartia, I. Bahl, and A. Ittipiboon, Microstrip Antenna Design Handbook, Artech House, Norwood, Mass, USA, 2001.

[11] L. R. Lewis, M. Fassett, and J. Hunt, "A broadband stripline array element," in Proceedings of IEEE International Symposium on Antennas Propagation Society, vol. 12, pp. 335-337, 1974.

[12] P. J. Gibson, “The Vivaldi aerial," in Proceedings of the European Microwave Conference, vol. 9, pp. 101-105, 1979.

[13] J. Shin and D. H. Schaubert, "A parameter study of striplinefed vivaldi notch-antenna arrays," IEEE Transactions on Antennas and Propagation, vol. 47, no. 5, pp. 879-886, 1999.

[14] T. H. Chio and D. H. Schaubert, "Parameter study and design of wide-band widescan dual-polarized tapered slot antenna arrays," IEEE Transactions on Antennas and Propagation, vol. 48, no. 6, pp. 879-886, 2000.

[15] D. M. Pozar, Microwave Engineering, John Wiley \& Sons, New York, NY, USA, 3rd edition, 2005. 

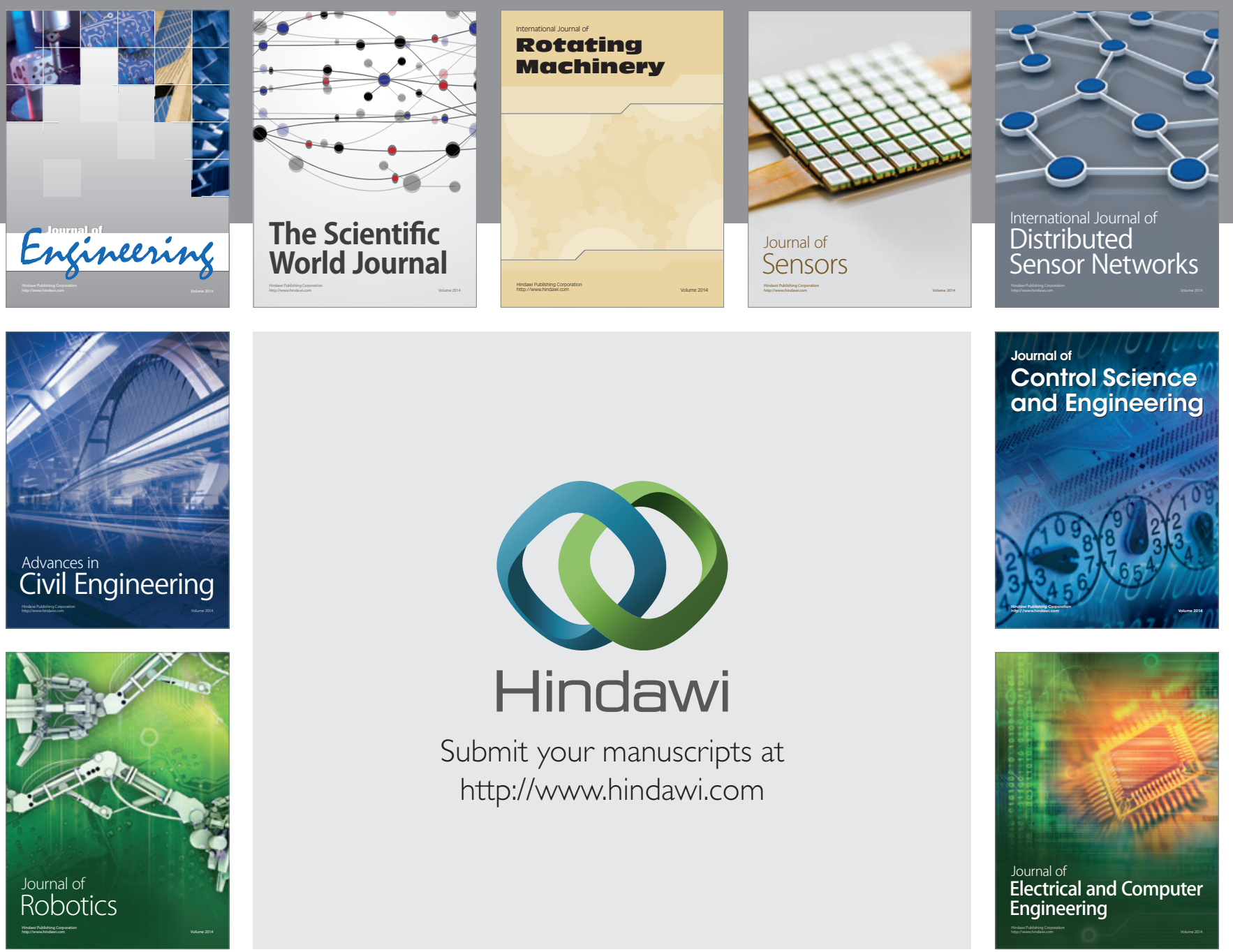

Submit your manuscripts at

http://www.hindawi.com
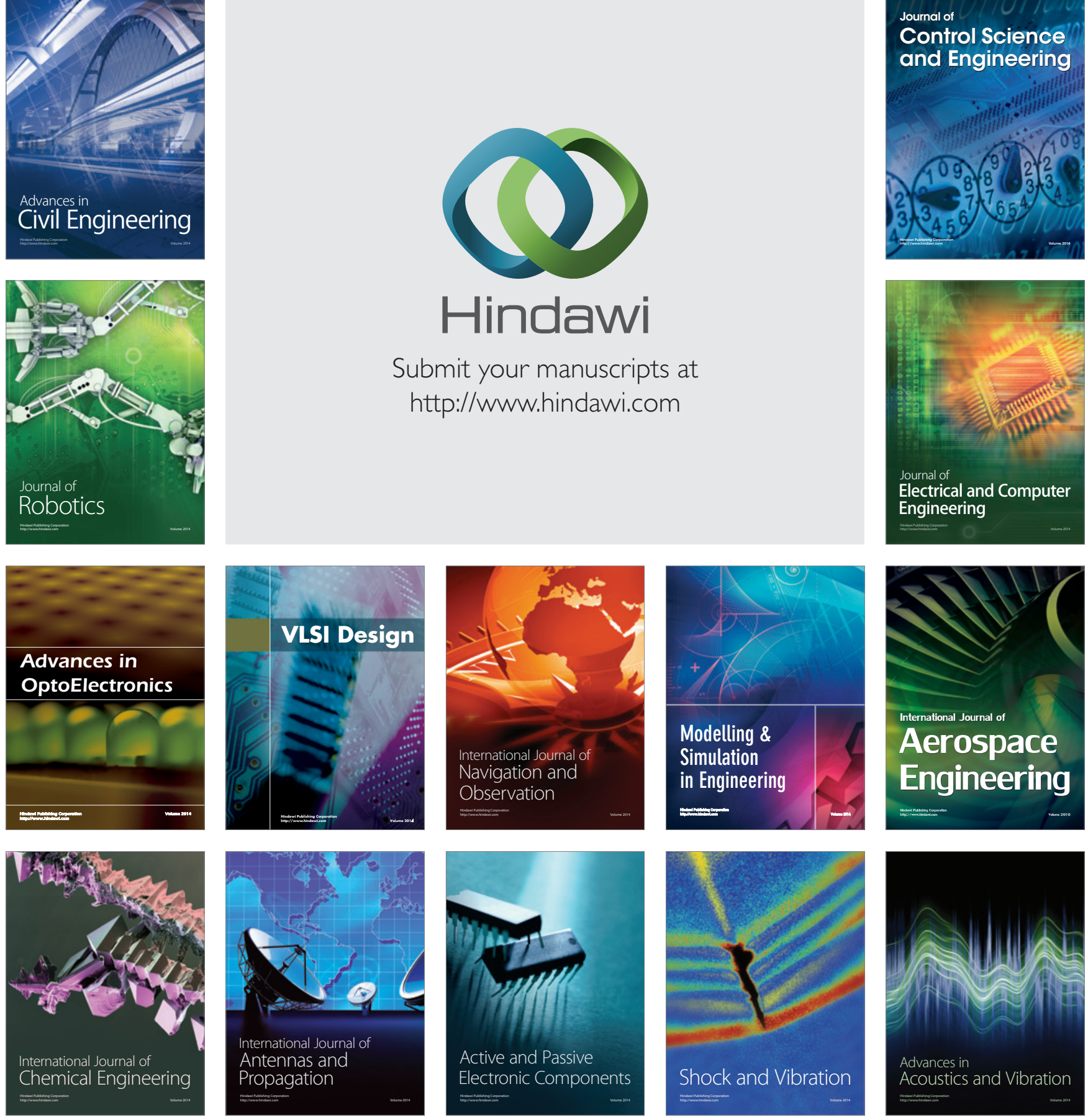